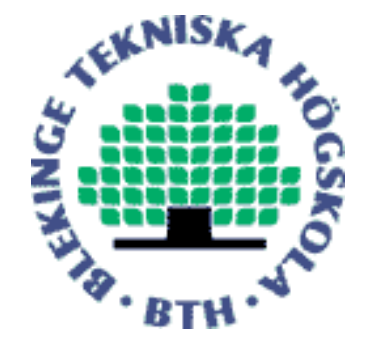

Copyright (C) 2011 IEEE.

Citation for the published paper:

Challenges and opportunities of sensor based user empowerment

Jenny Lundberg, Rune Gustavsson

8th IEEE International conference on sensor, networks and control (ICNSC)

2011 Delft, The Netherlands

This material is posted here with permission of the IEEE. Such permission of the IEEE does not in any way imply IEEE endorsement of any of BTH's products or services Internal or personal use of this material is permitted. However, permission to reprint/republish this material for advertising or promotional purposes or for creating new collective works for resale or redistribution must be obtained from the IEEE by sending a blank email message to pubs-permissions@iee.org.

By choosing to view this document, you agree to all provisions of the copyright laws protecting it. 


\title{
Challenges and opportunities of sensor based \\ User empowerment
}

\author{
Jenny Lundberg, Rune Gustavsson
}

\begin{abstract}
Sensor and sensor networks technology are becoming an increasingly important part of information management infrastructures. Challenges related to collection, processing, analysis and user-centric presentation depending of context are of particular interest. Furthermore, design and implementation of proper respond actions to sensor based information is gaining interest in many applications. In this paper we focus on robust application of sensor based systems. With robust we mean in this context supporting user empowerment and allowing adaptations. Two case studies of critical operations for individuals with cognitive impairments are presented. A comprehensive methodology supporting empowerment of users is outlined. The methodology includes ethnography-based analysis of workflows to identify user dependant barriers as a basis for design and implementation of mechanisms supporting empowerment. Identified challenges and opportunities of sensor based user empowerment is a contribution. Requirements engineering, from a user empowerments perspective including user specific barriers and sensor based information mechanisms, as a part of the systems developments process is another important contribution. Usercentric training, as an important validation of implemented solutions in given context, is part of lessons learned. Sections on challenges and opportunities as well as of future work conclude the paper.
\end{abstract}

\section{INTRODUCTION}

$\mathrm{R}$ OBUST information sharing and decision support tools based on sensor input can potentially provide a win-win situation with positive effects for the individual as well as opportunities for societal and economical gains.

We focus in this paper on sensor systems supporting user empowerment. The sensor systems envisioned aims at supporting users' traditional senses of sight, hearing, smell and tactile input to access, process and act purposeful in a given environment. The information processing to that end is only partly understood but different cognitive models such as the Belief-Desire-Intention (BDI) model have been used to model and implement different kinds of decision support system (DSS). Of particular interest to us is Multi-Agent technologies based on the BDI model, e.g., JACK [17].

By user empowerment we understand in this context individually tailored support systems aiming at supporting the users understanding the present context and to take proper actions. In particular we focus, in this paper, on users that have different kinds of cognitive impairments. Present

Manuscript received January 5, 2011

Jenny Lundberg is with Blekinge Institute of Technology, 37179 Karlskrona, Sweden, (phone:0455-385873; e-mail: jlu@bth.se).

Rune Gustavsson is with Blekinge Institute of Technology, 37179 Karlskrona, Sweden, (e-mail: rgu@bth.se). evolutions of sensor networks in Smart homes and in Health care are of particularly interest and usefulness for this large group of users [10][20][22][25].

The contemporary models and techniques of DSS, e.g., knowledge engineering meet a clear barrier when it comes to model and implement decision systems constrained by the capabilities of users with special needs.

We suggest and illustrate in this paper a methodology whereby design of proper sensor systems combined with user-centric evaluation and training can remedy some of the barriers related to different cognitive impairments in designing and implementing proper DSS in those contexts. Our methodology is a configuration of different methodologies under the umbrella of Participatory Design (PD). PD is a design approach attempting to involve all stakeholders in the design process where the main aim is usability.

\section{RELATED WORK}

Related work focusing on understanding and finding patterns of everyday tasks adding sensor input to DSS exist, however, usually the methodological approach typically do not include PD approaches [23][24] or lack focus on applications involving users with cognitive impairments [26]. Related work focusing on sensor based DSS towards persons with cognitive impairments exists [27]. However, work on PD approaches with an individually tailored focus towards semantically correct information sharing with validation and monitoring aspects are mainly absent at the moment.

\section{CASe StUdies}

We have conducted two case studies of people with special needs in their day-to-day living. The scientific studies were conducted by researchers from Blekinge Institute of Technology (BTH) specialized in computer science, knowledge engineering, ethnographical studies, interaction design, Participatory Design (PD) and Human Computer Interaction (HCI). Other team members were different professional teams from the community of Sölvesborg in Blekinge, Sweden and, most important, the end-users $\mathrm{M}$ and $\mathrm{P}$ (introduced below) together with their families and other important persons in their daily life.

Case 1 addresses a cognitive related problem of person $\mathrm{P}$ having Asbergers syndrome. Her dysfunction basically concerns obsessive compulsive behavior (OCB) [8]. Her OCB problem addressed is when she is getting stuck by the 
door repeatedly assuring herself that she has actually locked the door.

Case 2 addresses remotely synchronization of activities of a person $\mathrm{M}$. M has been diagnosed to have "easy to average cognitive impairments", and his main concern is catching the bus in time getting to work. His impairment concerns estimating time, planning and making predictions of how long time different tasks will take.

Persons $\mathrm{P}$ and $\mathrm{M}$ are living on their own in regular apartments with assisted living support. They are very concerned of their impairments (as expressed above) to handle daily activities. Their families and other support persons are also constantly worrying about their situations.

\section{TOWARDS SENSOR SUPPORTED EMPOWERMENT}

The following Figure 1 captures our generic architecture of Decision Support Systems (DSS) based on trusted information sharing supported by inputs from sensor networks and actions grounded in the physical environment.

The main system components are; Context model, Information processing artifact, Interfaces, and Sensor networks. The users of the system are denoted by Sender and Receiver and the whole system is situated in a Context.

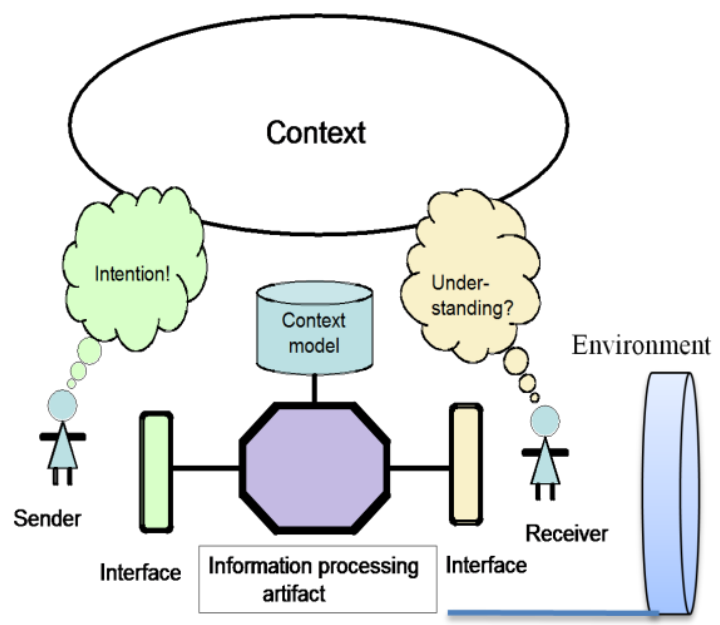

Figure 1. A generic architecture of sensor supported Decision Support systems empowering users.

The overall challenge of system analysis, design and implementation is to provide a semantically correct and trusted information exchange between the different users. We have addressed several aspects of these challenges elsewhere [12][13][14][15][16][19].

The specific challenge addressed in this paper is proper design and implementation of DSS when the user-receiver has cognitive impairments.

The suggested approach has cycles of activities including the following:

- Identification of workflows to be supported

- Identification of user specific barriers in following/understanding crucial steps of the workflow
- Identification of sensor based information mechanisms to empower the user

- $\quad$ Training and validation

The general idea is to empower the user with easy-tounderstand signs eliminating cognitive demanding reasoning, by users, that usually is the corner-stone of traditional knowledge based systems.

\section{TOWARDS A CONFIGURABLE METHODOLOGY}

Our approach is grounded in analysis of workflows aiming at capturing the intentions and barriers of users. The requirements process is based on Ethnography [1] and Participatory Design [2] approaches. The information perspective is grounded in Info Sense [4], built on Situation Theory [3]. Knowledge Engineering perspective has been built on CommonKADS methodology [28]. CommonKADS basically are a knowledge intensive methodology combining engineering and management. Design of empowerment mechanisms are based on interaction design capturing the intent of the user while harnessing the identified barriers. Implementation of the support mechanisms are based on principles of service oriented systems and sensor based mechanisms. Training assuring user acceptance of the solutions supports assessments, and is a part of the validations process.

The requirements and validation approach is supported by an iterative use of Boehm's spiral model [18] (CommonKADS) for software engineering development processes. Validations have been performed continuously during the processes, however the end implementation and validation in real world has not yet been performed. The validation is to be performed against workflow, basically performing their workflow activities. This since other approaches such as concept validations [33] is not suitable for the individuals with cognitive impairments in focus. This mainly for two reasons, since the persons with cognitive impairments may have difficulties in envisioning the concept, and that the acceptance process including training are of importance.

Similar configurations of methodologies are used in both cases; however, the outcomes differ since the solutions are domain and user dependent.

\section{FINDINGS}

Following our approach outlined in Section IV we have for our Case I with user P and Case II with user M.

\section{A. Case I Empowering P to leave locked door}

Modeling of critical workflows of situations from the user P's perspective: 
Lock door

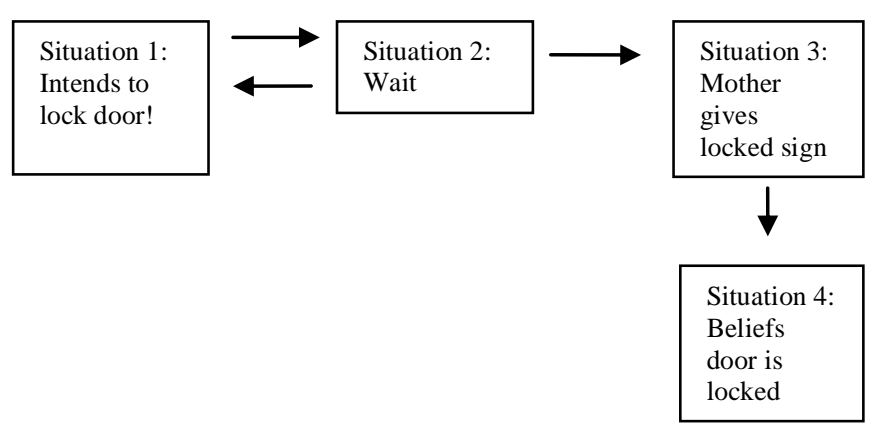

Figure 2. Situation and actions performed by $\mathrm{P}$.

In Figure 2 we illustrate the OCB by $\mathrm{P}$, leading to her getting stuck in repeatedly locking the door and then forgetting about this immediately after, unless she can be assured by observing the proper sign from her mother.

Our suggested solution is to use a "smart key" that is triggered by a signal from the lock when it is closed, Figure 3.

Lock door

Observe icon

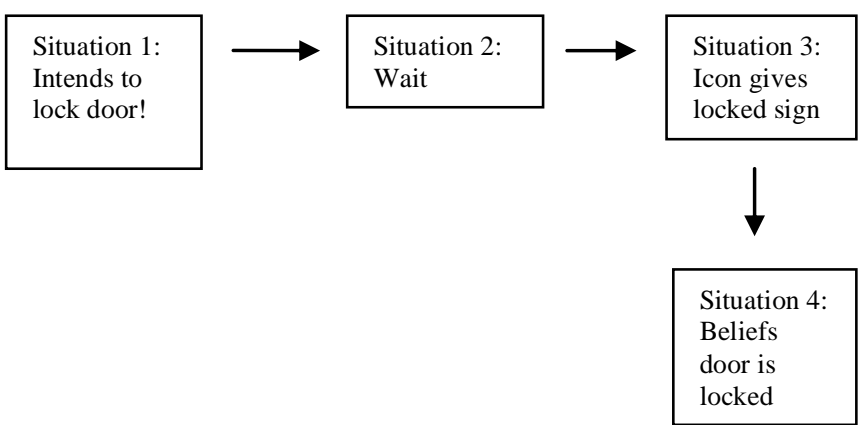

Figure 3. Situations and actions performed by an empowered $\mathrm{P}$.

The signal is represented as an icon showing that the door is locked. The icon is then replacing $\mathrm{P}$ 's mother as a confirmation, to $\mathrm{P}$, that the door is indeed locked. Of course, the trust in the icon has to be trained with $\mathrm{P}$ to be accepted and trusted by $\mathrm{P}$ as a valid substitute of her mother's sign.

As assurance system NumberedOutTime protocol, indicating too many re-locking of door under to short amount of time can be alerted for the support personnel.

\section{B. Case II Empowering $M$ to catch the bus in time}

In order to support $\mathrm{M}$ in his planning and doings, his support team has beforehand identified the different steps to be performed by $\mathrm{M}$ from waking up, preparing himself for the day, having breakfast, brushing teeth, picking up his money, mobile and leaving his apartment to get to the bus stop.

The durations of the different activities has been measured and sequenced. In order to help $\mathrm{M}$ the team has prepared a corresponding set of signals that were transmitted to his wrist-watch. When $M$ heard the clock signal he was supposed to look at the message and thus synchronize himself to perform remaining tasks and keep the time to get the right bus.

However, during our ethnographical studies of his workflows and the role of the reminders by the clock, our main findings were:

1. M routinely switched off the clock without looking at the message.

2. $\mathrm{M}$ was disturbed by the implemented monitoring and synchronization efforts

The good intentions of supporting $\mathrm{M}$ in his planning and execution of daily morning activities, by pre-defined remote monitoring and intervening, were in fact a failure and a frustration to $\mathrm{M}$.

Our approach of empowering $\mathrm{M}$ is based on identifying a 'critical path' in M's workflow activity that would trigger a signal from his activities to his support team. The support team could then estimate the time to the next bus departure and inform $\mathrm{M}$ about this by sending one suitable message to his mobile phone/information device.

The starting point of the 'critical path' has been identified as $\mathrm{M}$ picking up his mobile phone and wallet. Triggering of sensor is connected to this activity. Furthermore, a confirmation signal to the team can be sent when $M$ is closing his door leaving his department.

In short: Instead of remotely trying to remind $\mathrm{M}$ of the steps of an intended workflow we suggest that a well identified action (sensor based) should be triggered and sent to the support team and thus synchronize them to give relevant information to $\mathrm{M}$ "just in time" about departure time of his bus. Furthermore the team could estimate the time, i.e. the probability of catching the bus in connection to the signal of that $\mathrm{M}$ has left his apartment.

Our suggested solution will empower $M$ without unnecessary infringement of his integrity. An initial prototype with this empowerments approach, critical path based sensor - smartphone application has been engineered [32]. Again training by $\mathrm{M}$ is necessary to accept this sensorbased support.

\section{CHALLENGES AND OPPORTUNITIES}

The main contribution in our approach is proper identification of workflows including user-specific barriers and mechanisms supporting empowerment. Furthermore, the following challenges and opportunities of sensor-based empowerment have been identified:

\section{Challenges:}

- Semantic understanding of sensor based information sharing connected to situations.

- Monitoring and affordance of sensor input/output.

- Causal activities based on sensor input (Trust and complexity).

- Empowerment models. 
- Social aspects, roles and information exchange possibilities.

- Training and validation models.

Opportunities:

- Cost effective update of information and situation in comparison to constant monitoring.

- Flexibility of ambient support.

- Enhancement and distribution of information perspectives.

Creating robust information infrastructures individually tailored empowering persons with cognitive impairments built on sensor based DSS, these challenges and opportunities need to be taken into consideration. In [6] these issues are further elaborated. Based on our Participatory Design workflow approach the intention is the initiating and read thread during process.

Understanding the fundamentals of semantic for assuring correct information sharing, Info Sense, Devlin's logical framework [3][4] of structuring of information can preferably be seen as a high level description of information exchange. The connection between Information (understood, or interpreted, by a human agent) and its Representation is captured by the following equation:

\section{Information $=$ Representation + Interpretation}

The equation describe that information are visible via a representation. The representation could for example be a book, a computer system or similar. The Interpretation describes the interpretation capabilities of the receiving agent. Given this, we are aware of the emphasis that can be necessary to put on semantics.

Semantic understanding of sensor based information sharing can include implementation of ontologies based on the model of what situation the information are exchanged in, what the focal situation are and what the information means for each person in the situation in focus. After clarifying workflow, situation and context based relations, ontologies can be implemented. Basically semantics are seen as a relation not an object, figure 1. Knowledge models including cognitive impairments can be an important contribution in this process.

From a high abstractions perspective the situation [3] are a critical issue, thus in our approach the quality of understanding the situation are based on the sensor type and application. Suitable sensor types that disambiguate causal models are of importance. Robust connection of sensor input is created when relating the sensors to the situation framed by identified acts related to physical room and/or artifact. Given the situation base, robust identification of interaction models, data models and protocols is fundamentals and are considered.

The affordance given in the interface, in figure 1 , for the person with cognitive impairments sending signal to service person should be integrated in workflow. The affordance of the information artifact is sent by triggering of sensor (automated or by human agent) received by the persons with cognitive impairments is to be based on the impairment modeled in empowerments model where the ability, context and information required of the person are included.

Monitoring aspects including workflow of support persons are of importance. Being mobile, the sensor input alerts should be adapted to that specific situation. When using the mobile device the support persons usually are supporting other persons, so tactile, sound alerts and extended automation support could be suitable for those situations. When stationary the documentation and analysis support can be extended.

Training and validation approaches including the person to be empowered are necessary to perform against workflow preferably in cooperation with persons that are trustworthy, service personnel, parent, or other proper mediators to the new information application. Based on the performed iterative validations, reduction of complexity when describing the sensor system for the end users, i.e. is most probably necessary.

Sensor systems from a high-level abstraction are basically cause - effect relations. Modeling causality based reasoning [30] and controlling so it is stabled implemented in realworld following the model are critical issues. Trust issues are connected to causal reasoning, i.e. how to make predictions of effects based on functions i.e. human behavior based [11]

Agent solutions based on impairment, sensor input, sensor protocol, social inferences and causality models are reasonable. In the social aspects, data structures, interaction and data models based on 'street smart' social behavior can be of relevance [9][29][30]. It could include implementation aspects taking into consideration if several staff are available, which staff is most socially suitable and similar to the individual in focus.

\section{FUTURE WORK}

Future work has been initiated and will include implementation of the empowerments approach including the validation and training of prototype against workflow.

Resilience structures [31] are of importance and infrastructure perspectives including robust connectivity, energy sources, update and scalability issues are fundamentals. On software application level resilience are closely connected to protocol update from customer input based on Service Level Agreement (SLA) such as activating/deactivating and adding/removing sensors and based on that, coordination and combination between sensor network inputs and inferences. Cloud solutions where identification of suitable channels, and possible inferences between documents based on the support person's situation are reasonable.

Web 2.0 solutions [21] are to be considered in sensor systems based on this PD approaches since integrity and trust issues are possible to integrate and identify in the methodological approach [5] [11]. 


\section{CONCLuSiOnS}

The ongoing evolution of smart homes and related health care will be even more net- and knowledge based, Figure 1. The challenges of inclusion of people with special needs are key topics internationally. Not the least within EU. However, traditional models and methods for requirement engineering, system design and implementation have to be supplemented with methods and tools identifying user specific barriers (focus of empowerments) models and techniques understanding how sensor-based information can be transformed and utilized to support user empowerment.

In this paper we have illustrated some methodological issues related to two case studies. We have given solutions whereby users with cognitive impairments can be empowered by suitable sensor networks to handle their daily tasks in a way that respects their integrity. Challenges and opportunities of sensor based user empowerment are critical to consider towards robust sensor and sensor networks technology infrastructure.

\section{REFERENCES}

[1] Hammersly, M., Atkinson, P., Ethnography - principles in practice, Routledge London and New York, ISBN 0-415-08664-7, 1995

[2] Greenbaum, J. \& Kyng, M. (Eds.) Design at Work, pp. 169- 196. Hillsdale, New Jersey: Laurence Erlbaum Associates, 1991

[3] Barwise, J. and Perry J. Situations and attitudes. CSLI Publications, 1999

[4] Devlin, K, Info sense, W.H. Freeman and Company, New York, 2001

[5] Gustavsson, R., Lundberg, J., Rindebäck, C., Ådahl, K., Functional verses non-functional requirements considered harmful, World Congress in Computer Science, Computer Engineering and Applied Computing, Las Vegas, USA, 2007

[6] Lundberg, J. Engineering principles for open socio-technical systems, BTH Dissertation Series, School of Engineering, Blekinge Institute of Technology, ISBN:978-91-7295-183-9, 2011, to be defended $16^{\text {th }}$ of February

[7] Boehm, B., A spiral model of software development and enhancement, ACM SIGSOFT Software Engineering Notes", 11(4):14-24, August 1986

[8] http://www.internetmedicin.se/dyn_main.asp?page $=804$, $\mathrm{h}: 10.44$,

[9] Miller, J. H., Page, S. E. Complex adaptive systems - an introduction to computational models of social life, Princeton University Press, 2007

[10] Baker, N., Zafar, M., Moltchanov, B., Knappmeyer, M., ContextAware Systems and Implications for Future Internet, Towards the Future Internet, Tselentis, G., et al. (Eds.) IOS Press, doi:10.3233/9781-60750-007-0-335, 2009

[11] Rindebäck, C. and Gustavsson, R., Why Trust is Hard - Challenges in e-mediated Services. In Proceedings of Workshop on Trust in Agent Societies at the Conference Autonomous Agents and Multi Agent Systems 2004 (AAMAS-04). Special issue of LNAI on Trusting Agents for trusting Electronic Societies, pp. 180 - 199, Springer Verlag, ISBN: 978-3-540-28012-5., 2005

[12] Rindebäck, C. Designing and maintaining trustworthy online services. BTH licentiate dissertation series No. 2007:08, School of Engineering, Blekinge Institute of Technology, ISSN: 1650-2140., 2007

[13] Brandt, P., Information in Use -Aspects on Information quality in workflows, BTH Dissertation Series No. 2007: 04, School of Engineering, Blekinge Institute of Technology, ISSN 1653-2090, 2007
[14] Ådahl, K. Transparency of critical Information for patient empowerment in e-Health. BTH licentiate dissertation series No. 2007:03, School of Engineering, Blekinge Institute of Technology, ISBN: 978-91-7295-107-5, 2007

[15] Östlund, L. Information in Use - In- and Outsourcing Aspects on Digital Services. BTH Dissertation Series No. 2007:05, School of Engineering, Blekinge Institute of Technology, ISSN 1653-2090, 2007

[16] Lundberg, J., Principles of Workflow Support in Life Critical Situations. BTH licentiate dissertation series No. 2007:02, School of Engineering, Blekinge Institute of Technology, ISBN: 978-91-7295$105-1,2007$

[17] http://www.aosgrp.com/products/jack/index.html

[18] Boehm B, A spiral model of software development and enhancement, ACM SIGSOFT Software Engineering Notes", "ACM", 11(4):14-24, August 1986

[19] Lundberg, J., Gustavsson, R., Robust approach toward context dependant information sharing in distributed environment, The $11^{\text {th }}$ International conference on Enterprise Information Systems, Milan, Italy, 2009

[20] Piniewski, B., Muskens, J., Estevez, L., Carroll, R., Cnossen, R., Empowering health-care patients with smart technology, Computer, IEEE July 2010

[21] Domingo, M. C. Managing healthcare through social networks, Computer, IEEE July 2010

[22] Alarmnet Wireless sensor network for smart healthcare, http://www.cs.virginia.edu/wsn/medical/

[23] Lymberopoulos, D., Bamis A., and Savvides, A., Extracting spatiotemporal human activities patterns in assisted living using home sensor networks, PETRA, Athens, Greece, 2008

[24] Kawasaki, H., Ohmura, R., Osawa, H., Imai, M., A model for addition of user information to sensor data obtained from living environment, CASEMANS, ACM International Conference Proceeding Series, 2009

[25] Helal, S., Mann, W., El-Zabadani, H., King, J., Kaddoura, Y., Jansen, E., The Gator Tech Smart House: a programmable pervasive space Computer, v 38, n 3, p 50-60, March 2005

[26] Demirisa, G:, Oliverb, D., P., Dickeyc, M., S., Rantze M., Findings from a participatory evaluation of a smart home application for older adults, Technology and Health Care 16 111-118 111, IOS Press, 2008

[27] Chang., Y-J., Chang W., C., Wang, T-Y., Context-aware prompting to transition autonomously through vocational tasks for individuals with cognitive impairments, Assets '09: Proceedings of the 11th international ACM SIGACCESS conference on Computers and accessibility, 2009

[28] Schreiber, A., et al., Knowledge Engineering and Management: The CommonKADS Methodology. MIT Press., 2000

[29] Devlin, K., Goodbye Descartes, Wiley, 1998

[30] Pearl, J., Causality - Models, reasoning and inferences, Cambridge University Press, 2000

[31] Hollnagel, E.,Woods, D., D., and Leveson, N., Resilience Engineering: Concepts and Precepts, Ashgate Publishing, Aldershot Hampshire, UK, 2006

[32] http://tuba.bth.se/medieportal/play/224/Health-Intention

[33] Lee, L. M., Dey, A. K. Embedded assessment of aging adults - a concept validation with stakeholders, Pervasive Computing Technologies for Healthcare, Munich, Germany, IEEE publisher, 2010 